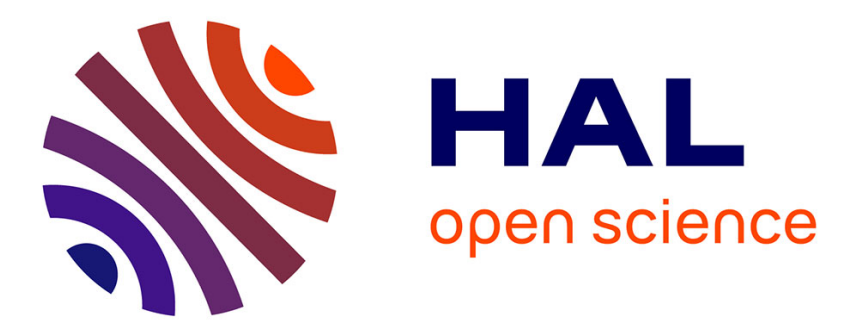

\title{
Reprises et détournements polémiques sur Twitter
}

Agata Jackiewicz

\section{To cite this version:}

Agata Jackiewicz. Reprises et détournements polémiques sur Twitter. Travaux de Linguistique: Revue Internationale de Linguistique Française, 2016, Les mèmes langagiers: propagation, figement et déformation, 73 (2), pp.85-105. 10.3917/tl.073.0085 . halshs-01838962

\section{HAL Id: halshs-01838962 \\ https://shs.hal.science/halshs-01838962}

Submitted on 13 Jul 2018

HAL is a multi-disciplinary open access archive for the deposit and dissemination of scientific research documents, whether they are published or not. The documents may come from teaching and research institutions in France or abroad, or from public or private research centers.
L'archive ouverte pluridisciplinaire HAL, est destinée au dépôt et à la diffusion de documents scientifiques de niveau recherche, publiés ou non, émanant des établissements d'enseignement et de recherche français ou étrangers, des laboratoires publics ou privés.

\section{(ㄷ)(1) $\$$}

Distributed under a Creative Commons Attribution - NonCommercial| 4.0 International 


\title{
REPRISES ET DÉTOURNEMENTS POLÉMIQUES SUR TWITTER
}

\author{
Agata JACKIEWICZ
}

\section{Introduction}

Les nouveaux dispositifs de communication numériques offrent des espaces d'expression et d'échange massivement investis par la société. Ces espaces médiatiques en ligne articulent différents niveaux d'interaction, de l'individu à ses cercles sociaux, du privé au public, de l'objet à ses référencements, reprises et commentaires. Ils induisent de nouvelles formes verbales et de nouveaux genres textuels de plus en plus complexes. Chaque production écrite ou orale (tweet, message sur Facebook, billet de blog, article de presse en ligne, vidéo...) est potentiellement commentée ou évaluée, citée ou référencée, reprise en l'état, enrichie ou détournée. Etant donnée la nature à la fois sociale, technologique et linguistique de ces discours et les problèmes d'explicitation et de formalisation que leur analyse implique, les chercheurs sont amenés à privilégier des cadres pluridisciplinaires et construire des outillages descriptifs diversifiés.

Notre projet général vise l'élaboration d'outils notionnels, linguistiques et informatiques permettant de proposer une lecture renouvelée de la conflictualité contemporaine et d'analyser la diversité de ses formes d'expression (Jackiewicz, 2016a, 2016b). L'étude présentée ici porte sur des représentations sociales et culturelles associées à la famille et à la filiation, telles qu'elles se manifestent lors des débats actuels sur le mariage homosexuel et la GPA (gestation pour autrui). Nous nous proposons d'éclairer les mécanismes de partage et de négociation critique de ces représentations en analysant leurs modes d'expression et de reprise dans des échanges sur la plate-forme Twitter.

* Praxiling, Université Paul-Valéry (Montpellier 3) ; 44 rue Raymond Losserand, 75014 Paris ; Agata.Jackiewicz@univ-montp3.fr 
Après avoir identifié les représentations associées au couple, à la famille et à la filiation qui se trouvent au cœur des négociations polémiques dans les corpus considérés, nous nous attachons à expliciter et à décrire les modes de reprise dialogique à l'œuvre dans des tweets qui en sont le support. Dans cette collection de tweets, circonscrite et catégorisée, nous identifions des unités récurrentes, pouvant apparaître sous forme stricte de segments répétés et sous forme détournée. Le défigement des unités témoigne en effet d'une stabilisation suffisante pour en faire des objets commucationnels autonomes et efficaces, «prêts pour la reprise ». Dotées d'une prégnance du signifiant (formes brèves, simples, binaires) et du signifié (contenu généralisant, doxal...), certaines d'entre elles (comme un papa et une maman, par exemple), fonctionnent comme des « formules », au sens de Krieg-Planque (2012).

La méthode ainsi définie défend l'idée que les faits de stabilisation en discours sont à rattacher à des usages précisément déterminés et situés. D'où l'importance accordée d'une part à l'analyse préalable des débats d'un point de vue sociétal qui explicite les représentations en jeu et d'autre part à l'exploration des corpus numériques qui reflètent ces débats. Ce sont ces deux exigences méthodologiques qui rendent ce travail consistant et le rapprochent de la perspective mémétique ${ }^{1}$, même si l'approche virale de la propagation des représentations (et des objets langagiers correspondants) n'est nullement opératoire ici. Nous n'expliquons pas pourquoi et comment ces représentations seraient «contagieuses », au sens de Dawkins (1978) ou de Sperber (1996).

Sur le plan linguistique, cette démarche conduit à discriminer trois ordres d'indices linguistiques qui renvoient respectivement au cadre éristique du débat (l'éristique étant 'l'art de la dispute et du débat'), aux formes spécifiques de dialogisme que ce cadre mobilise, ainsi qu' au caractère essentiellement normatif des contenus communiqués.

\section{Contexte scientifique}

\subsection{Bref état de l'art}

La question des propriétés caractéristiques et des modalités verbales de la délibération polémique a débouché sur de nombreux travaux en analyse du discours. Signalons tout d'abord les apports majeurs de KerbratOrecchioni (1980, 2005), Angenot (1982, 2008), Maingueneau (1983, 2008), ainsi que les recherches d'Amossy et de ses collaborateurs (Amossy et Burger, 2011 ; Koren, 2011 ; Amossy, 2014). Des études plus ciblées, portant sur certains aspects des échanges agoniques, comme la violence verbale (insulte...), la rationalité et l'irrationalité ou l'implication émotionnelle, sont également éclairantes (Plantin, 1997, 2011 ; Desmons et 
Paveau, 2008 ; Moise et al., 2008 ; Ogier, 2012...). La discussion sur la spécificité du débat polémique par rapport au débat argumentatif en général, entreprise notamment par Plantin (2003), constitue une autre technique pour faire ressortir ses traits les plus saillants. Sans aboutir à un consensus, les auteurs, ceux cités plus haut mais également Rennes (2007) et bien d'autres, proposent différentes configurations de traits, nombreux et relatifs à des réalités variées, tels que la polarisation et la dichotomisation des thèses antagonistes, la présence d'un proposant, d'un opposant et d'un tiers, la violence verbale, le recours à des paralogismes... On en retiendra que la notion de polémique renvoie à un phénomène sociolangagier multidimensionnel, dont les propriétés définitoires relèvent de plusieurs ordres différents (social, politique, psychologique, linguistique, discursif...).

L'accès à des données massives, issues de dispositifs numériques, permet aujourd'hui de poursuivre ces travaux, en bénéficiant des outillages informatiques et des apports disciplinaires plus larges. Le dispositif communicationnel et énonciatif de Twitter produit des conditions spécifiques d'échange polémique, différents de ceux qui sont habituellement analysés débats ou duels télévisés, séries de discours monogérés compilés et rapportés par des médias (Amossy, 2014). Dans la sphère du Web, les échanges polémiques sur des forums de discussion ou des pages de commentaires associés à des articles, se rapprochent des tweets par leur aspect polylogue et le caractère anonyme des participants, mais s'en s'éloignent par plusieurs points propres aux tweets comme : l'absence d'ancrage, l'absence de modération, l'ampleur de la participation, la forme courte, les pratiques d'adressage et de reprise massive.

Au confluent de la sociologie et des sciences de l'information et de la communication, des travaux récents ont porté spécifiquement sur des questions polémiques d'ordre sociétal telles qu'elles se manifestent sur les RSN (réseaux sociaux-numériques). À partir de l'étude des pétitions électroniques demandant l'arrêt du débat sur l'identité nationale (ou la suppression du ministère éponyme à son origine), Boure et Bousquet (2011) interrogent les façons contemporaines d'intervenir dans l'espace public et de mettre en visibilité une cause. À partir des observations menées sur Twitter, Cervulle et Pailler (2013) décrivent finement les lignes de force et l' "économie affective» des débats sur l'ouverture du mariage aux couples de personnes de même sexe. Julliard et Cervulle (2013) étudient la construction discursive de la «différence des sexes » telle qu'elle émerge des deux controverses sociétales portant respectivement sur la parité et «le mariage pour tous ». Sujette à d'importantes tensions épistémiques et discursives opposant les différentialistes et les universalistes, « la différence des sexes » demeure une catégorie de pensée qui structure l'espace public. 


\subsection{Corpus}

Les discours éristiques sur Twitter se distinguent par plusieurs caractéristiques remarquables ${ }^{2}$. Ils présentent un ancrage contextuel significatif, une dimension intertextuelle et un dialogisme développé. La forme courte du tweet conduit les internautes à dire l'essentiel en peu de mots, sous une forme directe et synthétique. La densité en information y est corrélée à une structuration prégnante de son signifiant. Les opinions défendues et les arguments avancés sont exprimés dans des schémas d'expression simples et stéréotypés (propices à la stabilisation, comme nous le verrons dans les paragraphes qui suivent). Nous voyons dans les énoncés polémiques de Twitter des modèles élémentaires de verbalisations qui peuvent composer des discours polémiques plus élaborés.

Notre corpus d'observables est constitué de plusieurs collections de tweets, en rapport avec un ensemble de sujets polémiques touchant au mariage, à la procréation et à la filiation. Ces questions sont de nature à trouver un très large écho dans la société civile et donc sur l'ensemble des dispositifs de communication qu'elle emploie.

Deux corpus de tweets seront exploités préférentiellement pour cette étude. Le premier est relatif à l'ouverture du mariage aux couples de personnes de même sexe (corpus «MAR»). Le deuxième concerne le débat sur la filiation issue de la GPA (corpus «FIL »). Les tweets sont datés pour permettre d'adosser l'analyse aux événements qui ont constitué les temps forts de ces deux controverses (débats, auditions à l'AN, manifestations nationales...). Le corpus «FIL », notre corpus principal, a été collecté le 5 octobre 2014, durant 24 heures, à partir des mots-clés suivants : pma, gpa, procréation pour autrui, gestation pour autrui, filiation, famille, manif pour tous, manifpourtous. Il comprend au total 184300 tweets. En simplifiant, la mobilisation organisée ce jour servait à montrer l'opposition à la GPA et installer dans l'espace public la cause d'une filiation «naturelle », vécue comme menacée par le récent accès au mariage accordé aux couples homosexuels.

Selon le généticien Axel Kahn (2010 : 146-147), « la gestation pour autrui, c'est la pratique de la mère porteuse, technique qui permet de porter un enfant en dehors de tout rapport charnel, à partir des ovocytes d'une autre femme. [...] Cette technique d'insémination artificielle remet en cause une règle fondamentale du droit de filiation. En droit français, la mère est la femme qui accouche 'mater semper certa est' (ou plutôt 'mater certissima, pater semper incertus'?). Si le père n'est pas certain, la mère l'est ». La controverse éthique qui en découle porte sur l'intérêt de l'enfant à naître, le risque d'exploitation des femmes, la marchandisation des corps, l'atteinte à leur dignité. 


\section{Représentations de la famille et de la filiation}

Nous allons nous intéresser maintenant aux éléments représentationnels associés à la famille et à la filiation, tels qu'ils émergent des analyses des corpus constitués. Comme nous le verrons, la question des normes y est omniprésente.

\subsection{Représentations sociales et culturelles : quelques repères}

Les représentations sociales et culturelles sont des systèmes d'idées construits et entretenus collectivement qui s'intercalent en quelque sorte entre le monde et la compréhension que l'on en a. Ces représentations touchent à l'organisation sociale, à l'imaginaire collectif, aux systèmes symboliques. Historiquement construites, elles présentent une certaine permanence. On y trouve des valeurs profondément ancrées et transmises d'une génération à l'autre, qui forment leur héritage culturel. Elles constituent un facteur de cohésion et de différenciation, selon que les individus adhèrent à des éléments communs ou différents. Les représentations sociales et culturelles passent par des phases de construction et de renforcement, mais connaissent également des moments d'affaiblissement ou de déstructuration. Pour une présentation plus ample de ce concept, le lecteur pourra consulter les travaux fondateurs de Jodelet (1984) ou Moscovici (1986).

\subsection{Mutations de la famille, diversification des modèles}

Si l'évolution et la diversification des paysages familiaux, attestées depuis un demi-siècle au moins dans les sociétés occidentales, constituent aujourd'hui une évidence, les récents débats sur le mariage homosexuel et la gestation pour autrui ont réactivé la question du statut de la famille et des liens de filiation. La mutation des schémas familiaux (familles monoparentales, recomposées, homoparentales...) entraine celle de la filiation. À l'évidence, ce n'est plus la procréation seule qui fait le parent (Théry, 2013). La conception actuelle de la filiation est tiraillée entre deux pôles : une représentation naturaliste fondée sur l'engendrement et le lien de sang (filiation restreinte) et une valorisation des liens librement choisis (liens du cœur) et l'expression des sentiments (filiation élargie).

Le débat sur la famille et la filiation, tel qu'il apparaît dans nos corpus, fait ressortir plusieurs éléments remarquables. La question des normes (installées, bafouées, en évolution...) y est très présente. Les défenseurs affichés de la famille mettent en avant ses fondements biologiques [1-5].

[1] \#bourdindirect Une famille homo vit en dehors des normes de la reproduction humaine donc au sens biologique ce n'est pas une famille 
[2] Desceller entièrement la filiation de l'ordre naturel sous prétexte de son injustice signifie ouvrir grand la porte à l'eugénisme de masse.

[3] La \#GPA est une entrave à la nature. \#ManifDeLaHonte

[4] «Je viens défendre la famille naturelle. Toutes les civilisations se sont effondrées dans l'homosexualité, la Grèce

[5] Il y a des dérives qui font que des hommes croient aimer des hommes, pareil pour les femmes, mais leur amour ne peut pas former une famille.

Pour certains, les mutations qui affectent la famille sont autant de facteurs de son affaiblissement. Face à ce danger, on s'efforce à rappeler que (i) la famille, c'est la base / sacré / une forcel essentiel / tout ce qui compte/ primordial.... (ii) la famille, y'a que ça de vrai / y'a rien de mieux / avant tout... (iii) la famille est une valeur en soi / chère / refuge / cardinale / fondatrice / qui monte... On dit non à la destruction / décomposition / disparition de la famille. On appelle à la protéger, défendre ou sauver. L'expression valeur famille, récurrente dans les argumentaires de ses défenseurs, devient un étendard qui réunit implicitement tout ce que les normes installées imposent à cette construction sociale ${ }^{3}$.

[6] http://t.co/SINLmRf7aR @ valerieboyer13 Bravo pour votre mobilisation ainsi qu'au pape pour sauver la Valeur Famille http://t.co/ RTGxsEgJHK

[7]@elsa_hst oui mais pas dans ces extrémités quand même! C'est juste que la valeur famille a une très grande signification pour moi

[8] « la famille est la valeur refuge à protéger en priorité »

[9] « La \#Famille écosystème de base est à protéger en priorité »@XX \#FamillesEnAvant \#5oct @XX

[10] La valeur famille, Sans aucun doute, En Simca 1000 Ça tient la route. Pas de dérapage Je reste sage.\#MPT http://t.co/ZpbouuVEXS

Les voix favorables à la GPA s'opposent, quant à elles, à l'enfermement des humains dans des distinctions naturelles et à l'institutionnalisation du biologique [11-12]. Elles doutent de la supériorité du modèle traditionnel, allant jusqu'à moquer ses configurations accoutumées [13]. Des témoignages évoquant des situations familiales moins typiques ou hors norme, mais vécues comme heureuses, se font entendre [14-15]. L'évolution des modèles familiaux prouverait qu'il s'agit d'une construction sociale [16-18].

[11] Mais fichtre, qu'est-ce donc que la \#filiation ? Il existe encore des gens qui croient que le lien du sang est une valeur en soi ?

[12] «La filiation ne peut plus se réduire au fait procréatif »@DBertinotti, ex ministre de la famille \#PS\#manifpourtous \#GPA \#PMA

[13] Un papa + une maitresse + une maman + un curé + 12 enfants + une Renault Espace = une famille normale \#manifpourtous 
[14] Ma famille a 3 pères ou 0 des enfants, des demi-enfants ou pas, des cousins, des ancêtres, des amis et une cave a vin. Mais pas de normes.

[15] J'ai été éduqué dans une famille monoparentale. Je suis homosexuelle. Et je vais très bien. C'est quoi votre problème? \#LaManifPourTous

[16] Faudrait peut-être se reveiller, on est en 2014 et la famille à déjà bien évolué !! \#droitspourtous \#manifdelahonte

[17] Le \#SaviezVous : La notion de famille c'est comme la notion de fidélité, nous n'avons encore rien compris. \#Construit sociétal

[18] La famille c'est une institution, c'est culturel. On peut la faire évoluer, non ? Enfin c'est dur pour les simplets surtout.

\subsection{Bataille des représentations}

Dans l'esprit des défenseurs de la famille traditionnelle, le couple parental, procréateur, asymétrique et hétérogène, implique une différence des sexes, naturelle et universelle. Mais, pour d'autres, seuls les liens de cour (amour, attachement...) sont nécessaires pour en fonder une. Enfin, pour d'autres encore le principe de l'égalité suffit pour traiter et accepter de la même manière les divers schémas familiaux rencontrés. Nous sommes ainsi en présence de plusieurs systèmes normatifs que nous détaillerons dans les paragraphes qui suivent.

\subsection{1. « Un papa et une maman »?}

Sérieux, humoristiques, ironiques ou carrément caustiques, de nombreux tweets qui composent nos corpus renvoient à l'architecture de la famille. Au cœur de ces messages, plusieurs variantes de segments ciblant le couple parental (un papa et une maman ${ }^{4}$, un père+une mère, \#unpapaunemaman, \#unhommeunefemme) montrent que le débat se focalise sur la nature hétérogène et asymétrique du noyau familial. Ces HT ont donné lieu à des détournements polémiques (\#UnMixerUneCafetière, \#UnConUneConne...). Outre la blague, très populaire sur Internet, dont le patron («Un papa + une maman + ...») sert à exhiber les «attributs type » d'une famille « idéale » (13), voir aussi le $\$ 4.4$, de nombreux messages pointent les problèmes que pose une telle acception de la famille.

[19] «Famille = papa + maman » et les familles monoparentales ? Parce qu'un des parents est décédés ou autre, ils ne sont plus une famille?

[20] c'est vrai que c'est mieux de ne pas avoir de parents plutôt que deux du même sexe... \#ManifDeLaHonte

[21] C'est une vision tlmnt chrétienne des choses, « une famille c'est un homme une femme et des gosses ». On est en 2014 il serait temps d'évoluer 
Le recours à des références bibliques a été amplement utilisé dans ce débat, comme en témoignent les tweets ci-dessous. Le choix des éléments fondateurs de la religion catholique, mais destinés ici à soutenir la GPA, entend mettre les adversaires de cette option, en majorité catholiques, devant leurs contradictions et ébranler leurs argumentaires. Le fait de reprendre une même idée dans des formats d'expression très proches, simples et empreints d'humour, rapproche les tweets [23-32] des mèmes Internet $^{5}$.

[22] En tant que catholique, je suis pour la GPA puisque c'est comme ça que Jésus est né.

[23] \#ManifPourTous un papa, une maman. comme Jésus. euh... non, lui c'est pas un bon exemple. enfin si mais là non, mais euh... oh merde.

[24] \#UnPapaUneMaman: Pauvre Jésus, né d'un père inconnu Par ProcurationMiraculeuseAssistée, de nos jours ça ne serais plus admissible !

[25] Jésus, Marie, Joseph: une famille recomposée?http://t.co/ MTYLcjJgGr\#GPA

[26] Jésus : deux papas !Marie : PMA !

[27] Jésus : deux papas et une maman vierge. \#ManifDeLaHonte

[28] Jésus avait 12 apôtres et pas de femme, ça pourrait faire réfléchir certains cathos réacs;) \#ManifDeLaHonte \#ManifDesRéacs

[29] Jésus avait 2 papas \& 1 maman \& disait «aimez-vous les 1 les autres ». Ils ont compris qq chose \#LMPT ? \#homophobie \#racisme \#ManifDeLaHonte

[30] Jésus il avait bien 2 pères pourtant non ? Pffff.Comment peut-on souhaiter le malheur de certaines personnes ?!\#ManifDeLaHonte

[31] Jésus il est bien issu d'une GPA et il a deux papas, non? \#JaiToutCompris?

[32] Jésus, c'est pas le mec qui est né d'une mère porteuse et qui avait deux papas? \#ManifPourTous

Outre le texte, les images intégrées aux tweets peuvent véhiculer des représentations de la famille. Schématiques, souvent caricaturales, elles exploitent des stéréotypes et dévoilent les éléments que l'imaginaire collectif retient comme essentiels ou constitutifs d'une famille typique ou idéale. Différents procédés sont à l'œuvre : recyclage des images de propagande nataliste nazie, transformation et détournement de logos, recontextualisation de photos... Une simple recontextualisation d'un contenu iconique peut produire un effet de défigement ${ }^{6}$. 
Image 1 : Exemples des éléments iconiques intégrés aux tweets, qui renvoient à des représentations de la famille


Le vrai logo de la Manif Pour Tous
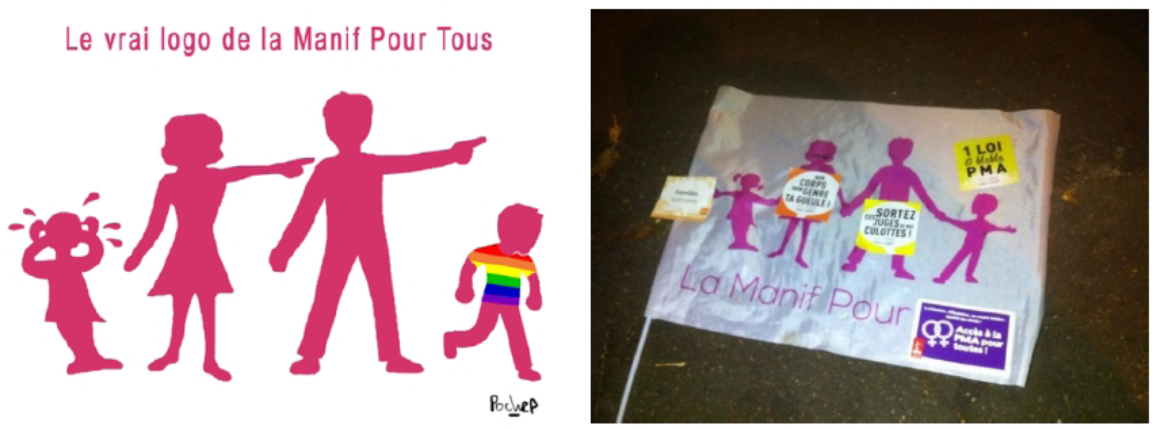

Certaines de ces images ont fait l'objet de nombreux tweets et retweets, avec des commentaires variés. Par exemple, on trouvera respectivement pour les quatre images données ci-dessus :

[33] Voici la véritable image de la famille que flamby souhaite détruire ! Honte à lui ! \#LMPT

[34] Exactement l'image qui résume la \#ManifPourTous\#ManifPourRien \#ManifDeLaHonte\#ManifDesCons

[35] La vrai logo de la Manif Pour Tous ${ }^{7}$

[36] La famille idéale revue et corrigée par le \#lobbyLGBT \#commandoLGBTpropreté

\subsection{2. « C'est contre nature »}

Si une famille traditionnelle sert de cadre pour une maternité et une filiation perçues comme naturelles, l'union entre personnes de même sexe et la pratique de la GPA sont qualifiées par d'aucuns de contre nature. Le jugement «X/c'est est contre nature » est à la fois un jugement de fait et un jugement de valeur. Il qualifie et condamne la pratique de $\mathrm{X}$, en raison du fait qu'elle serait absente de la nature, contraire à l'ordre naturel, ou 
a minima contraire à une norme communément acceptée. De nombreux échanges de tweets focalisent le débat sur cet obstacle à l'acceptation des configurations familiales qui échappent aux normes établies.

[37] Le mariage homo c'est contre nature

[38] \#MariageGay ouvre une spirale infernale dans le droit et dans la société ! Un mariage contre nature ! \#TeamValeurs fondamentales !

[39] J'suis contre le mariage gay pcq j'ai ma religion ect mais aussi excusé moi mais c'est contre nature.

[40] Le mariage gay je suis contre! Si Allah a crée un homme et une femme c'est pas pour rien . Rien de plus contre nature !\#Homo

[41] Moi j'suis contre le mariage Homo parce que selon moi c'est contre nature, et puis quoi encore ? Dans 20 ans mariagae homme-animal ? \#Stop

La réfutation de ce point de vue peut prendre différentes formes. Certains pointent sa non-pertinence pour la question soulevée. D'autres retournent l'argument en l'appliquant à l'attitude de leurs détenteurs.

[42] On peut être contre le mariage homo et manifester contre. Mais dire que c'est « contre nature » c'est complètement stupide.

[43] C'est pas notre Amour qui est contre nature, c'est votre putain d'imbécilité. \#ManifPourTous

[44] Ce qui est contre nature c'est de se battre contre le droit des autres. \#ManifPourTous \#ManifDeLaHonte

[45] Christine Boutin est anti mariage gay car c'est contre nature mais elle est marié à son cousin germain. Et l'inceste c'est normal ?

Le refus de la nécessité biologique de l'hétérogénéité des couples se fait également entendre.

[46] \#ManifDeLaHonte l'homosexualité est tellement contre nature qu'elle existe chez quasiment toutes les espèces animales, pas l'homophobie

[47] \#ManifDeLaHonte \#lamanifpourrie. Les lionnes elevent les petits entre elles. Contre nature?

[48] A ceux qui disent que le mariage homo est contre nature, je les rassure : le mariage hétéro ne l'est pas non plus. \#ManifDeLaHonte

Contrer l'argument en allant sur le terrain de l'adversaire constitue un mode de subversion permettant de mettre en lumière les contradictions dans son raisonnement : pousser sa logique jusqu'au bout, tourner l'argument en dérision... Plus généralement, recourir à l'ironie ou à la provocation (enfin, $l o l \ldots)$ ou plus simplement répondre par l'humour ou le bon mot constituent les options souvent choisies par des twittos. Il est à remarquer que le détournement de la formule contre nature dans [52-54] n'est interprétable que si l'on a connaissance de la teneur du débat en cours. 
[49] Si Dieu est le créateur de toute chose, il est créateur de l'homosexualité. Dieu serait-il contre nature ?\#LMPT\#ManifDeLaHonte

[50] Nous faire croire qu'un mariage homo est contre nature alors qu' une vierge enceinte de Dieu pas de soucis... \#manifpourtous

[51] L'homosexualité est contre nature. C'est vrai que marcher sur l'eau, changer l'eau en vin et ressusciter c'est naturel. \#MariagePourTous

[52] Si l'on y réfléchit, s'habiller est également un choix contre nature. A POIL LES VERSAILLAIS! A POIL ! \#ManifDeLaHonte

[53] \#ManifDeLaHonte des hommes en robe c'est pas contre nature? http://t.co/ss3umvdt43

[54] M'en fous moi demain je vais manifester contre les choux de Bruxelles... Je trouve ça contre nature \#Manifpourtous

\subsection{3. \#Amourpourtous, \#égalitépourtous}

En réponse à la mobilisation contre la GPA et le mariage homosexuel, de nombreuses personnes ont choisi d'avancer un seul argument : l'amour. Cette attitude repose sur la valorisation des liens librement choisis (les liens du cœur) et l'expression des sentiments.

[55] Pour moi la famille, c'est l'amour. Ça n'a rien de légal et ça n'a rien de biologique.

[56] \#NotInMyName \#amourpourtous \#ManifDeLaHonte

[57] En ce lundi matin, les «valeurs de la famille» sont les mêmes qu'hier : l'amour, l'amour, l'amour. Bonne journée à tous.

[58] Lafamille=Amour, Partage, et ouverture d'esprit\#ManifPourlEgalite \#MurCitoyen

Pour aller dans le même sens, d'autres ont préféré l'argument de l'égalité. La valorisation de l'égalité implique l'abolition de la hiérarchie familiale, traditionnellement basée sur la distinction masculin-féminin.

[59] Ok argument invalide mais là on parle de valeur, d'un principe fondateur de la RF : l'égalité. \# lrt \# ManifPourTous

[60] Sérieusement manifester contre l'égalité. il y a de quoi avoir honte. \# ManifDeLaHonte

[61] «Liberté, Egalité, Fraternité », mais que pour les blancs hétéro catholiques c'est ça ? \#ManifDeLaHonte

[62] La logique des anti-égalité de la \#ManifPourTous

\section{Procédés linguistiques}

Dans les ensembles de tweets que nous venons de rapporter, nous attestons, en simplifiant, de deux grandes familles de modes d'expression : (i) des structures simples (X être/avoir Y...) et (ii) des structures composites 
construites sur différents rapports d'interdiscursivité et de dialogisme. Nous allons explorer plus en profondeur la deuxième de ces familles.

Dans une interaction polémique, discours et contre-discours s'articulent au moyen des stratégies de reprises discursives, réinterprétations, concessions, réfutations, négations... Des fragments de discours autres - sous forme de référence, citation, allusion, reformulation, négation, détournement... - sont potentiellement présents dans chacune des interventions constituantes. Ces procédés de négociation sont propices à véhiculer des représentations qui se trouvent au cœur des débats d'idées.

\subsection{La citation}

En situation de controverse, les discours respectifs sont repris et interprétés au cours de l'interaction verbale et souvent «adaptés » en fonction des besoins des interlocuteurs.

Dans le corpus «FIL », les tweets de citation et les retweets à l'identique (ou presque) sont un moyen simple de signaler l'adhésion à une parole jugée pertinente, tout en en augmentant l'audience et l'impact. Certains tweets détaillent l'origine ou le parcours d'une citation.

[63] RT @Maitre_Eolas: En tant que catholique, je suis pour la GPA puisque c'est comme ça que Jésus est né.

[64] «On instrumentalise la famille pour promouvoir un modèle religieux »@benoithamon à propos de @LaManifPourTous sur@ franceinter. Bien dit!

[65] «La filiation ne peut plus se réduire au fait procréatif » @ DBertinotti, ex ministre de la famille \#PS\#manifpourtous \#GPA \#PMA

[66] «Un enfant sur quatre est élevé par un seul des deux parents : la famille a évolué, il faut en tenir compte»@danycohnbendit \#E1matin

Un compte spécifique @_ilsOntDit (« les perles de nos politiques sont ici ») retwitte des propos des hommes et femmes politiques. Il est à noter que les citations reprises dans ce débat sont souvent celles qui évoquent les différents principes fondateurs de la famille.

[67] RT @_ilsOntDit: «Il est temps de s'affranchir de cette définition naturaliste de la famille. » @ najatvb http://t.co/n7ikPIwVz6

[68] RT @_ilsOntDit: « Il faut dissocier la sexualité de la parentalité et la famille de son strict rapport au biologique »@ meuniermichelle PS

[69] RT@_ilsOntDit: «Lafamilleestuneconstruction socialecrééeparles hommes pour dominer les femmes »@R_Bachelot\#manifpourtous

[70] RT @_ilsOntDit: «La filiation ne peut plus se réduire au fait procréatif »@DBertinotti, ex ministre de la famille \#PS\#manifpourtous \#GP... 
[71] RT @_ilsOntDit: « la loi sur le \#mariagepourtous sort la famille du fantasme un papa une maman et un enfant »@meuniermichelle http://t.co/kQ...

[72] RT @_ilsOntDit: Ce qui pose problème c'est cette famille idéalisée, HÉTÉRO-PATRIARCALE-BLANCHE@meuniermichelle PS \#familiephobie http://t...

La circulation des propos polémiques est génératrice de commentaires négatifs, de transformations importantes, voire d'oublis volontaires, l'objectif étant de questionner, de disqualifier ou de falsifier la parole de l'autre. Le désaccord a besoin d'un signalement explicite.

[73] « La non-discrimination n'est pas l'indifférenciation » Fou comme les convictions se périment vite \#ManifPourTous \#5oct

[74] «Famille = papa + maman » et les familles monoparentales ? Parce qu'un des parents est décédés ou autre, ils ne sont plus une famille?

[75] «La famille est le socle de notre société.» La TOLERANCE est le socle de toutes les sociétés, pauvre chiard. \#LMPT

[76] «@Bouffie_: J'ai deux mamans, et j'en suis fière. \#ManifPourRien \#ManifDeLaHonte » C'est grave sale

Dans le corpus «FIL », de nombreux tweets sont postés en réaction à une affirmation sentencieuse ou un slogan, entendus lors de la manifestation ou lus dans des médias. Une négation polémique ( «la femme n’est pas X », « La France a besoin de $\mathrm{X}$, pas de $\mathrm{Y}$ »...) peut être reprise et attaquée à son tour, comme dans [77-82].

[77] « La femme n'est pas une machine à bébés », dit cette militante de la \#ManifPourTous mère de 5 enfants.

[78] « La femme n'est pas une usine à bébé! ». Dixit une femme interviewée lors de la \#ManifPourTous. Précision, elle a déclaré avoir 9 enfants...

[79] «La France a besoin d'enfants, pas d'homosexuels » Omg j'ai honte. \#ManifDeLaHonte

[80] « La France a besoin d'enfants, pas d'homosexuels » et les enfants gays? les homos qui veulent des enfants ?!\#ManifDeLaHonte \#ManifPourTous

[81] « la france a besoin d'enfant pas d'homo » qui vous dit que les enfant sont contre les gays? \#ManifDeLaHonte

[82] «LA FRANCEA BESOIN D'HUMAIN PAS D'HOMOPHOBES » c'est une bonne réponse a leur banderole \#ManifDeLaHonte

\subsection{Négation polémique}

Dans un contexte polémique, la négation donne à voir un contre-discours, tout en s'employant en même temps à le combattre. Ses trois modalités 
particulières, les réfutations par négation, par retournement et par dénégation, ont pour point commun qu' ' elles repoussent un discours dont simultanément elles portent l'inscription » (Krieg-Planque, 2012: 210). Ces procédés apparaissent souvent dans des énoncés porteurs de contreanalyses.

La réfutation par négation présente une structure ordinaire « X n'est pas $\mathrm{P} »$, par laquelle elle réfute une affirmation qui est ou aurait été faite par l'adversaire.

[83] L'humain n'est pas une marchandise : Ni PMA, ni GPA ! \#5oct \#ManifPourTous

[84] L'humain n'est pas un objet! Pour ces pauvres femmes qui seront exploitées et risqueront la mort pour le désir de qq riches... NON À LA \#GPA

[85] \#LMPT L'enfant n'est pas un bien de consommation au profit de qq'un \#GPA \#PMA jamais

La réfutation par retournement obéit au schéma « c'est $X$ que $P$ et ce n'est pas $\mathrm{Y}$ que $\mathrm{P} »$ comportant une focalisation (une forme d'emphase).

[86] C'est Adam et Eve, pas Adam et Adam. \#ManifPourTous

[87] contre le mariage homosexuel Dieu a crée Adam \& Eve pas Benoit et Thomas !

La réfutation par dénégation quant à elle peut être formulée comme suit : «ce n'est pas $\mathrm{Y}$ que $\mathrm{P}$ mais $\mathrm{X}$ que $\mathrm{P}$ », où $\mathrm{Y}$ est soumis en même temps à la focalisation et à la négation.

[88] C'est pas l'homosexualité qui est une maladie mais plutôt l'homophobie. \#ManifDeLaHonte

[89] L'homosexualité n'est pas un choix. L'homophobie, en est un. \#ManifDeLaHonte

[90] La GPA contrairement au mariage n'est pas l'accomplissement d'un combat pour l'égalité, c'est juste l'extension de la sphère marchande.

[91] Contrairement à l'intolérance, l'amour n'est pas dangereux. \#mariagegay \#ouiaumariagegay

\subsection{Reformulation interdiscursive polémique}

Dans une interaction conflictuelle, un mode particulier de reprise de discours consiste en une reformulation polémique. Cette opération permet au locuteur de remettre en question une première saisie d'un état de chose et d'en proposer une autre (Steuckardt, 2007 : 4). D'un point de vue pragmatique, une reformulation interdiscursive polémique constitue un procédé de rectification ou de dévoilement. 
Selon les cas, l'énoncé comportera la séquence complète (X, en fait/ en réalité c'est $\mathrm{Y}$ ), ou seulement le segment reformulant (en fait/réalité c'est $\mathrm{Y}$ ). Les marqueurs en fait et en réalité présentent la première assertion comme non conforme à la réalité, à l'inverse de la seconde. Cette première assertion n'est pas nécessairement (complètement) exprimée, elle peut être déduite de la seconde. C'est le cas de la majorité des tweets, qui reformulent polémiquement des énoncés lus ou entendus quelque part. Un motif récurrent employé dans nos corpus de tweets semble aller dans le même sens : «ce qui est... c'est... ». Il permet de réfuter un argument implicite, car présent et saillant dans la mémoire des débatteurs, par exemple celui qui a été exprimé sur Twitter ou vu sur des pancartes des manifestants.

[92] En réalité, la \#ManifPourTous n'a qu'une volonté : imposer sa religion à la France. Ce temps là est fini, et heureusement.

[93] Ce qui est contre nature c'est de se battre contre le droit des autres. \#ManifPourTous \#ManifDeLaHonte

[94] Ce qui est contre nature, c'est d'aller a l'encontre des droits d'une personne en fonction de son orientation sexuelle! \#ManifDeLaHonte

\subsection{Détournement}

Tout énoncé faisant partie du patrimoine culturel commun, et plus généralement tout contenu qui relève d'un préconstruit, est susceptible d'être repris et recyclé, par exemple défigé, aussi bien sur un mode plaisant qu'ironique. À l'évidence, l'existence d'un rapport de sens avec le sujet traité augmentera l'impact de la formule trouvée. Une référence biblique (Adam et Eve), un titre de film (Trois hommes et un couffin) ou une phrase issue d'un jeu populaire (Dans la famille... je demande...), par exemple, auront d'autant plus de chances d'être détournés qu'ils renvoient à l'origine au domaine concerné par le débat (ici, la famille). Mais inversement, adopter un style ou un genre de discours fortement connoté et décalé par rapport au sujet traité (par exemple, un contrat de vente en viager) peut servir à frapper l'imagination en pointant des risques insoupçonnés (celui d'une dérive marchande...).

[95] C'est Adam et Eve, pas Adam et Adam. \#ManifPourTous

[96] au cinéma : « 2 hommes et un couffin » à la \#ManifDeLaHonte : «deux connes et une \#Boutin»

[97] dans la famille SousLaTerrasse, je voudrais le père-pioche! \#7FamilleDeLigonnès ${ }^{8}$

[98] La \#GPA est un viager gestationel où la mère porteuse n'a que la nue propriété de son enfant jusqu'à ce que s'exerce l'usufruit du payeur. 
Différents modes de déformation et de défigement sont à l'œuvre dans des interactions à caractère polémique. Formellement, la transformation peut consister en une fusion, une substitution, une réduction, un déplacement, une expansion, une réduction, pour reprendre la typologie de Villers (2010). Avec \#cestlaluttenuptiale nous sommes en présence d'une particitation militante au sens de Maingueneau (2012), obtenue par substitution (finale à nuptiale). La substitution de syllabe (comète à gamète dans Envisager la GPA, c'est faire des plans sur la gamète) ou de mot (trois à deux dans Deux hommes et un couffin) avec une ressemblance formelle ou catégorielle optimale et des relations de sens aisément interprétables fait partie des mécanismes transformationnels les plus courants.

La question de l'égalité devant la loi a amené plusieurs participants au débat sur la GPA à reprendre dans leur argumentaire la devise républicaine «liberté, égalité, fraternité» ou l'article 1 de la Déclaration des Droits de l'Homme et du Citoyen «Les hommes naissent et demeurent libres et égaux en droits ». Par un simple ajout d'une expansion, il s'agit de dénoncer sur un mode ironique l'existence d'exceptions dans l'application de ces principes.

[99] Ceux qui défilent à la manif pour tous veulent que les hommes soient « égaux sans triques »

[100] les hommes naissent et demeurent libres et égaux en droits, sauf s'ils sont homosexuels, autant pour moi \#ManifDeLaHonte

[101] «Liberté, Egalité, Fraternité », mais que pour les blancs hétéro catholiques c'est ça? \#ManifDeLaHonte

Un autre procédé qui mérite l'attention est le détournement des sigles. Cette pratique consiste à substituer d'autres mots commençant par les mêmes lettres à ceux qui composent le sigle original développé. Comme le fait remarquer Renard (2011:1), cette créativité langagière prétend le plus souvent à l'humour, mais elle se donne aussi pour objectif de dénoncer la « face cachée » de l'objet désigné par le sigle. L'auteur identifie quatre types de détournement selon leur finalité dominante : ludique, injurieux, antithétique et dévoilant.

Le détournement des sigles (GPA, PMA) attesté dans le corpus FIL possède une teneur polémique, sans véhiculer systématiquement une attitude hostile aux réalités ainsi désignées.

[102] GPA = Gestation Pour Abandon mais PARDON??? \#manifpourtous \#manifpourhomophobes

[103] GPA.GrandPrixde l'Amour

[104] \#UnPapaUneMaman: Pauvre Jésus, né d'un père inconnu Par ProcurationMiraculeuseAssistée, de nos jours ça ne serais plus admissible ! 
Le détournement des définitions, et notamment celles que l'un des protagonistes donne aux entités qu'il entend protéger (ici la famille : « La famille c'est un père et une mère »), correspond globalement à la même logique. La dérision et le dévoilement des faces sombres des familles traditionnelles sont clairement présents dans les tweets ci-après.

[105] La famille idéale c'est : un papa une maman, un cheval un mouton

[106] Vous avez rien compris. La famille idéale c'est : deux papas, une maman, un âne et un bœuf.

[107] Une famille c'est un papa, une maman, des enfants et un congélateur.

Les cas de détournement immédiat de mots-dièse (HT) sont nombreux ${ }^{9}$. On constate une forte créativité qui amène à reprendre (et à travestir) certains mots-dièse devenus populaires, pour polémiquer ou au contraire pour soutenir un message. Dans le corpus «FIL », les mots-dièse les plus fréquents désignent les organisateurs de la manifestation du 5 octobre 2014. À partir du nom et du mot dièse correspondant \#ManifPourTous de nombreux HT de forme \#manifXX ont vu le jour: \#manifPloufPlouf, \#manifcouscous, \#manifcontreautrui, \#manifcontretous, \#manifdelahaine, \#manifdelahonte, \#manifdecons, \#manifdecrétins, \#manifdesglands, \#manifdemes2, \#manifpourcons, \#manifpourhomophobes, \#manifpourpresquetous, \#manifpourlegalité, \#manifpourlaliberté... Le passage de \#manifpourtous à \#manifpourpersonne (\#manifpourrien, \#manifpourpresquetous...), par exemple, correspond à un détournement antithétique, par retournement, pour faire signifier à ce deuxième terme le contraire de ce que signifie ou évoque le premier.

[108] Essayez donc d'être heureux avant de passer votre temps nous rendre malheureux. \#ManifPourTous \#ManifPourPersonne

Dans le corpus «MAR », l'expression XXpourtous, engendrée à partir de la formule mariage pour tous (devenue en mot-dièse \#mariagepourtous, avec des variantes \#MariagePourTous...) prend 230 formes différentes (\#divorcepourtous, \#adoptionpourtous...) et apparaît dans 38000 occurrences. Le corpus FIL fait apparaître une orientation spécifique privilégiant, pour ce qui concerne les partisans de la PMA et la GPA, l'usage des mots-dièse qui renvoient à l'égalité (\#droitspourtous, \#droitalafamillepourtous, \#egalitepourtous) et aux émotions ou attitudes sociales positives (\#amourpourtous, \#respectdetous, \#espoirpourtous).

[109] Faudrait peut-être se reveiller, on est en 2014 et la famille à déjà bien évolué !! \#droitspourtous \#manifdelahonte

[110] France. Octobre 2014. Encore une occasion de perdre espoir en la nature humaine. \#ManifDeLaHonte \#droitalafamillepourtous 
[111] \#NotInMyName \#amourpourtous \#ManifDeLaHonte

[112] Il est ou l'\#espoirpourtous, le \#respectdetous ?! \#ManifDeLaHonte

\section{Discussion}

Les débats polémiques autour du mariage homosexuel et de la GPA ont placé au cœur des négociations plusieurs configurations familiales, dont les fondements sont vus comme relevant des principes différents : biologique ou naturel, affectif, égalitaire.

D'un point de vue linguistique, les discours qui font circuler sur Twitter les éléments représentationnels correspondants sont repérables par des configurations de marques qui se répartissent sur trois niveaux de signalement. Le fil de ces débats est indexé principalement par la récurrence des mots dièse \#mariagepourtous et \#manifpourtous et de leurs variantes polémiques ou humoristiques (\#Xpourtous, \#manifX). Les différentes stratégies de reprise discursive (négation polémique, reformulation interdiscursive polémique, citation, détournement...), avec leurs marquages spécifiques, permettent de cibler les contenus qui sont fortement débattus. Ces contenus comportent à leur tour des segments récurrents lesquels renvoient à des principes normatifs (X est contre nature, $X$ est une construction sociale, $X$ est une valeur en soi, un $X$ et un $Y$, valeur $X, X$ idéal/idéalisé, \#droitàX...).

Certains d'entre eux, comme un papa et une maman (\#UnpapaUnemaman...), cumulent plusieurs propriétés les rendant saillants : brièveté, format syntaxique simple, caractère binaire, assertion nominale, autonomie référentielle, caractère généralisant, contenu doxal (capacité à condenser des valeurs), prégnance cognitive, commentaires métadiscursifs ${ }^{10}$. Conjuguant ainsi la «bonne forme » et un contenu à prétention de vérité essentielle, de telles expressions relèvent du régime de surassertion qui favorise leur circulation et partage. Pour cette raison, tout segment dérivé (un papa et un papa, un papa + une maîtresse + une maman + ..., 2 papas \& 1 maman, deux papas et une maman vierge...) sera interprété dans le cadre de ce débat au regard de l'expression originelle, immédiatement identifiée.

La réflexion que nous venons de livrer tend à montrer que l'analyse de la délibération polémique permet de faire apparaître des phénomènes de stabilisation (temporaire ou durable) des unités discursives qui servent de support à des représentations sociales et culturelles.

De manière plus immédiate, ce travail entend contribuer à la description de la matérialité linguistique des controverses sociétales. Il fournit des outils d'analyse pouvant aider à la compréhension des représentations socioculturelles et des valeurs axiologiques mobilisées autour des notions choisies (ici « famille » et « filiation »). 


\section{NOTES}

1. Qui s'intéresse à la diffusion rapide et massive des objets culturels sur Internet

2. Pour une présentation générale du dispositif de Twitter, le lecteur pourra consulter entre autres : Stenger et Coutant (2011), Barton et Lee (2013), Jackiewicz et Vidak (2014) et Paveau (2013, 2015).

3. Ce qui motive par ailleurs ses reprises humoristiques ou ironiques, comme dans [10]. L'association texte - image dans ce tweet joue clairement sur les stéréotypes du genre, comme on peut le voir sur l'image associée (https://twitter.com/ VertuVirilite/status/518742526166327296/photo/1).

4. Rappelons que le slogan «Un papa. Une maman. On ne ment pas aux enfants » est devenu une formulation phare des manifestants anti-mariage homosexuel dès octobre 2012. Au même moment, une vidéo portant le même message circule sur Internet (voir : http://lelab.europe1.fr/video-mans-mariage-adoptioncouples-homosexuels-un-papa-une-maman-alliance-vita-5374). Destinée à créer le Buzz et qualifiée de virale par les médias, la vidéo montre un happening de l'association Alliance Vita, dont la scénographie (un homme-oiseau dont les ailes droite et gauche portent respectivement les inscriptions PAPA et MAMAN) est sensée démontrer qu'un enfant ne peut pas 's'envoler' s'il n'a pas un père et une mère.

5. Cet anglicisme désigne des «contenus auto-réplicatifs du Web» (images, textes, vidéos) à visée humoristique, et plus généralement tout élément ou phénomène repris et décliné en masse sur Internet https://fr.wikipedia.org/wiki/Mème_ Internet.

6. Cette question reste à approfondir dans une étude de plus grande ampleur sur les rapports texte-image dans des objets communicationnels circulant sur Internet.

7. Le traitement qui a été réservé au logo du collectif la Manif pour tous fait penser aux mèmes parodiques d'Internet.

8. À la référence au Jeu des 7 familles se rajoute ici l'allusion à l'affaire Dupont de Ligonnès (dite aussi « la tuerie de Nantes »).

9. Ces comportements sont spécifiques des débats ayant pour support une plateforme numérique.

10. Exprimés souvent en dehors de Twitter: une formulation phare, une blague qui fait fureur... Voir : http://www.huffingtonpost.fr/2013/01/13/mariagegay-blague-papa-maman-twitter_n_2465950.html

\section{RÉFÉRENCES BIBLIOGRAPHIQUES}

Amossy R. et Burger M. (éds), 2011, «Polémiques médiatiques et journalistiques. Le discours polémique en questions », Semen, 31.

Amossy R., 2014, Apologie de la polémique, Paris, PUF.

Angenot M., 1982, La parole pamphlétaire. Typologie des discours modernes, Paris, Payot. 
Angenot M., 2008, Le dialogue de sourd. Traité de rhétorique antilogique, Paris, Mille et une Nuits.

Barton D. et Lee C., 2013, Language Online. Investigating Digital Texts and Practices, Routledge Google books.

Boure R. et Bousquet F., 2011, « La construction polyphonique des pétitions en ligne. Le cas des appels contre le débat sur l'identité nationale », Questions de communication [En ligne], 20 | 2011, URL : http://questionsdecommunication.revues.org/2120.

Cervulle M. et Pailler F., 2014, «\#mariagepourtous : Twitter et la politique affective des hashtags », Revue française des sciences de l'information et de la communication, 4, http://rfsic.revues.org/717.

Dawkins R., 1978, Le gène égoïste, Paris, Editions Menges.

Desmons E. et Paveau M.-A. (éds) 2008, Outrages, insultes, blasphèmes et injures : violences du langage et polices du discours, Paris, L'Harmattan.

JACKIEWICZ A., 2016a, «Matérialité linguistique des controverses sociétales. Rapports intersubjectifs et interdiscursifs dans des tweets polémiques », CMLF, Tours.

JACKIEWICZ A., 2016b, Études sur les discours évaluatifs et d'opinion, Paris, L'Harmattan.

JACKIEWICZ A. et VIDAK M., 2014, «Étude sur les mots-dièse », CMLF, Berlin.

Jodelet D., 1984, «Représentations sociales : phénomènes, concepts et théorie », in Moscovici S., Psychologie sociale, Paris, PUF, p. 357-378.

Julliard V. et Cervulle M., 2013, «'Différence des sexes' et controverses médiatiques : du débat sur la parité au "mariage pour tous" 1998-2013 », Le Temps des médias, 21, p. 161-175.

KaHn A., 2010, Un type bien ne fait pas ça... Morale, éthique et itinéraire personnel, Paris, NIL.

Kerbrat-Orecchioni C., 1980, « La polémique et ses définitions », in Gelas N., Kerbrat-Orecchioni C., Le discours polémique, p. 3-40.

Kerbrat-Orecchioni C., 2005, Le discours en interaction, Paris, A. Colin.

KoRen R., 2011, «De la rationalité et/ou de l'irrationalité des polémiqueurs : Certitudes et incertitudes », Semen, 31, URL : http://semen.revues.org/9061

Krieg-Planque A., 2012, Analyser les discours institutionnels, A. Colin, Paris.

Maingueneau D., 1983, Sémantique de la polémique, Lausanne, L'Age d'Homme.

Maingueneau D., 2008, «Les trois dimensions du polémique », in GaudiNBordes L. et SAlvan G., Les registres. Enjeux stylistiques et visées pragmatiques, 11, p. 109-120.

Maingueneau D., 2012, Les phrases sans texte, Paris, Armand Colin.

Moise C., Auger N., Fracchiolla B. et Schultz-Romain Ch. (éds), 2008, «La violence verbale », vol. 1 et 2, Espaces politiques et médiatiques, Paris, L'Harmattan.

Moscovici S., 1986, «L'ère des représentations sociales », in Doise W., Palmonari A., L'études des représentations sociales, Neuchâtel / Paris, Delachaux \& Niestlé.

OGIER C., 2012, «La conflictualité en discours : le recours à l'injure dans les arènes publiques ». Argumentation et Analyse du Discours [En ligne], 8, URL : http://aad.revues.org/1297. 
Paveau M.-A., 2013, « Genre de discours et technologie discursive. Tweet, twittécriture et twittérature », Pratiques 157-158, p. 7-30.

Paveau M.-A., 2015, « Ce qui s'écrit dans les univers numériques », Itinéraires [En ligne], 2014-1।2015, URL : http://itineraires.revues.org/2313 ; DOI : 10.4000/itineraires. 2313

Plantin Ch., 1997, « L'argumentation dans l'émotion », Pratiques, 96, p. 81-100.

Plantin Ch., 2003, «Des polémistes aux polémiqueurs », in Declerce G., Murat M. et Dangel J., La parole polémique, H. Champion, p. 377-408, $<$ halshs-00425283>.

Plantin CH., 2011, Les bonnes raisons des émotions. Principes et méthode pour l'étude du discours émotionné, Bern, Peter Lang.

Rennes J., 2007, «Analyser une controverse. De la science politique à l'étude argumentative » in Bonnafous S. et M. Temmar M., Analyse de discours et sciences humaines et sociales, Paris, Ophrys, p. 91-107.

RENARD J.-B., 2011, « Le détournement de sigles. Entre jeu de mots et expression contestataire », Mots. Les langages du politique [En ligne], 95 | 2011, URL : http://mots.revues.org/20052

Sperber D., 1996, La contagion des idées, Paris, Odile Jacob.

Stenger T. et Coutant A. (éds), 2011, Ces réseaux numériques dits sociaux, Hermès, 59.

SteuckardT A., 2007, «Usages polémiques de la reformulation », Recherches linguistiques, 29, p. 55-74.

ThÉry I., éd., 2013, Mariage de même sexe et filiation, Paris, Éd. de l'EHESS.

Villers D., 2010, «Les modalités du détournement proverbial : entre contraintes et libertés », Modèles linguistiques [En ligne], 62|2010, URL : http:// ml.revues.org/237. 